\title{
Tecnura
}

http://revistas.udistrital.edu.co/ojs/index.php/Tecnura/issue/view/640

DOI: http://dx.doi.org/10.14483/udistrital.jour.tecnura.2014.SE1.a18

ReFLeXIÓN

\section{Accesibilidad territorial: instrumento de planificación urbana y regional}

\section{Territorial accessibillity: instrument for urban and regional planning}

\author{
Diego Alexander Escobar García*, Carlos Felipe Urazán Bonells**
}

\begin{abstract}
Citation / Para citar este artículo: Escobar García, D. A., \& Urazán Bonells, C. F. (2014). Accesibilidad territorial: instrumento de planificación urbana y regional. Revista Tecnura, Edición especial, 241-253.
\end{abstract}

Fecha de recepción: 29 de noviembre de 2013 / Fecha de aceptación: 11 de julio de 2014

\section{Resumen}

En esta investigación se explora el estado del arte respecto a los análisis de accesibilidad territorial y el uso de los mismos como instrumentos de planificación a nivel mundial. Se muestra claramente la metodología general de aplicación y la amplia gama de posibilidades existentes como un potente instrumento de planificación territorial a nivel urbano y regional. A lo largo del artículo se abordan ejemplos que muestran cómo la aplicación de esta metodología puede potenciar la toma de decisiones respecto a un proyecto de intervención futura, o reflejar necesidades en los cambios operativos de un servicio de transporte. Este instrumento de planificación se soporta en el procesamiento y análisis geoestadístico de una gran cantidad de datos, en su gran mayoría datos de posicionamiento satelital, características operativas, topológicas y geográficas de las redes que se va a estudiar. Para ambos niveles de planificación territorial se obtienen resultados gráficos valiosos que permiten identificar el impacto de las intervenciones o de los cambios operativos sobre el territorio. Este artículo muestra las bondades de la metodología como instrumento de planificación y concluye sobre la aplicación de la misma.

\begin{abstract}
This research explores the state of the art regarding territorial accessibility analysis and their use as planning tools worldwide. It clearly shows the general methodology of implementation, and the wide range of possibilities as a powerful tool for territorial planning in urban and regional level. Throughout the article we explore examples that show how the application of this methodology can enhance decision-making processes regarding a future intervention project, or reflecting needs for operational changes in a transport system. This planning tool is supported in processing and geostatistical analysis of a large amount of data, mostly, satellite positioning data, operational, geographical and topological characteristics of the networks studied. For both levels of territorial planning the graphic results show valuable maps in which the impact of interventions or operational changes over the territory can be identified. This article shows the advantages of this methodology as a planning tool and concludes about its implementation.
\end{abstract}

Keywords: accessibility, coverage analysis, GPS, impact evaluation, operability, planning tools.

\footnotetext{
* Ingeniero civil, doctor en gestión del territorio e infraestructura del transporte. Docente investigador del Departamento de Ingeniería Civil de la Universidad Nacional de Colombia. Manizales, Colombia. Contacto: daescobarga@unal.edu.co

** Ingenierio civil, doctor en gestión del territorio e infraestructura del transporte. Docente investigador del Departamento de Ingeniería Civil de la Universidad de La Salle. Bogotá, Colombia. Contacto: caurazan@unisalle.edu.co
} 


\section{INTRODUCCIÓN}

Las investigaciones relacionadas con los análisis de accesibilidad toman los conceptos fundamentales de la teoría de redes fundamentándose en métodos cuantitativos y geoespaciales (geoestadística), los cuales permiten obtener resultados gráficos bastante relevantes sobre las variables en estudio.

La planificación territorial, tanto a nivel urbano como regional, durante los últimos cincuenta años ha dado considerable importancia al concepto de "accesibilidad"; no obstante, su origen se remonta a la década de 1920 cuando se aplicó en áreas tan puntuales como la teoría de la localización y el planeamiento económico regional (Batty, 2009), convirtiéndose en un elemento primario de planificación urbana, mediante el cual se establecían criterios cuantitativos para la determinación del futuro uso del suelo, con lo cual se buscaba lograr un mayor bienestar social mediante la adecuada planificación sectorial (Kibambe Lubamba, Radoux, \& Defourny, 2013). Cuando se logra determinar que un territorio ofrece una deficiente accesibilidad a la comunidad, mediante la comparación de algunos estándares, es necesario el uso de herramientas investigativas que determinen los aspectos críticos de por qué dicha deficiencia, lo cual es fundamental en la adopción de políticas públicas, práctica que ha ido obteniendo cada día una mayor aceptación (Farrington \& Farrington, 2005).

La accesibilidad desde el punto de vista de teoría de redes, se define como la facilidad de comunicación que existe entre asentamientos humanos o actividades, la cual puede variar según el modo de transporte que se use (Morris, Dumble, \& Wigan, 1978) (Zhu \& Liu, 2004). Los modelos clásicos de accesibilidad establecen como principales variables la atracción entre los nodos que se interconectan en la red y la distancia existente entre los mismos (Curl, Nelson, \& Anable, 2011); se ha encontrado que esta medida es tan representativa que se debe entender como una necesidad secundaria no percibida (Halden, 2011), la cual finalmente no se establece como un objetivo sino como una condición indispensable de la red para permitir alcanzar eventos prioritarios para los habitantes, como, por ejemplo, educación, empleo, atención en salud, cultura, ocio, etc. Esta medida posee un valor no solo económico sino social ya que las personas y en ocasiones ciertas compañías aprecian tener adecuados niveles de accesibilidad, lo cual se traduce en una mejor calidad de vida y desarrollo económico (Gutierrez, 1998).

Desde el punto de vista urbano, se ha encontrado que una importante cantidad de países han enfocado sus esfuerzos en materia de transporte en eliminar las diferencias de clases sociales, mediante el ofrecimiento de un mayor acceso a los servicios y artículos de primera necesidad (Jones, 2011), para lo cual la reestructuración o evolución del modo de transporte público colectivo urbano, TPCU, influye en forma directa en la calidad de vida de los habitantes. Así mismo, a nivel urbano se han abordado investigaciones que involucran este concepto en campos como: acceso a parques públicos (Wang, Brown, \& Mateo-Babiano, 2013), cohesión social (Schürmann, Spiekermann, \& Wegener, 1997) (López, Gutiérrez, \& Gómez, 2008), sostenibilidad (Cheng, Bertoliniand, \& Clercq, 2007) (Vega, 2011), localización y prestación de servicios (Calcuttawala, 2006) (Park, 2012) (Higgs, Langford, \& Fry, 2013), demografía (Kotavaara, Antikainenand, \& Rusanen, 2011). A nivel regional, se han abordado investigaciones en campos como: análisis de cobertura (Straatemeier, 2008) y desarrollo económico (Rietveld \& Nijkamp, 1993) (Vickerman, Spiekermann, \& Wegener, 1999) (Mackinnon, Pirie, \& Gather, 2008).

Actualmente es posible afirmar que una de las variables por las cuales la accesibilidad en un territorio es baja es una deficiencia en la oferta de infraestructura vial (Burkey, 2012); no obstante, se ha encontrado que la accesibilidad está hoy día menos relacionada con la distancia que haya entre dos sitios, relacionándose en mayor proporción con la distancia que existe a las infraestructuras o modos de transporte y de cómo estas infraestructuras acortan los tiempos de conexión (Gutiérrez \& Gómez, 
The impact of orbital motorways on intra-metropolitan accessibility: the case of Madrid's M-40, 1999).

En Colombia aún no se ha dado el verdadero valor que posee un análisis de accesibilidad territorial; no obstante, ya se tienen algunos ejemplos reales de aplicación de estas metodologías en nuestro país, tanto a nivel regional (Escobar, García, \& Tolosa, Análisis de accesibilidad territorial a nivel regional, 2013) como urbano (Escobar \& García, Territorial Accessibility Analysis as a Key Variable for Diagnosis of Urban Mobility: A case study Manizzales (Colombia), 2012) (Escobar \& García, Diagnóstico de la movilidad urbana de Manizales, 2012) (Escobar \& García, Análisis de Priorización de proyectos viales, 2012) (Escobar \& García, Análisis de accesibilidad a nodos de actividad en Manizales (Colombia), 2012). Es necesario resaltar que los análisis de accesibilidad se han abordado hasta en temas relacionados con el turismo (Kastenholz, Eusébio, Figueiredo, \& Lima, 2012) y las redes sociales (Sailer, Marmot, \& Penn, 2012). No obstante, esta metodología está tomando una posición importante en la evaluación de planes y proyectos de infraestructura (Gutiérrez, Condeco-Melhorado, \& Martín, Using Accessibility indicators and GIS to assess spatial spillovers of transport infrastruture investments, 2012).

A continuación se repasan los aspectos metodológicos más importantes abordados en un análisis de accesibilidad y se muestran los pasos consecutivos que se llevan a cabo en el desarrollo de la metodología.

\section{METODOLOGÍA}

La metodología del análisis de accesibilidad, en general consta de cinco etapas consecutivas, las cuales se abordan a continuación.

\section{Puesta a punto de la red de infraestructuras y definición de escala de análisis}

En esta etapa inicial se busca actualizar completamente la red de infraestructuras que es objeto de estudio; mediante dicho procedimiento se establece también la escala del análisis, regional o urbano. Las fotografías aéreas y los mismos datos que se obtienen mediante los equipos GPS apoyan fuertemente esta etapa de la investigación.

\section{Obtención de las características operativas de la red de infraestructuras}

Para esta etapa se instalan equipos GPS en los diferentes modos de transporte relacionados con la red de infraestructuras, con el objeto de almacenar datos de posicionamiento satelital, que en conjunto aportan información básica sobre la operatividad de dicho modo.

\section{Procesamiento de datos obtenidos por los equipos GPS}

El procesamiento de las bases de datos requirió la aplicación de diferentes algoritmos de cálculo, con el objeto de establecer la velocidad de operación de cada uno de los arcos que componen la red de infraestructuras. Se analizaron tres parámetros en forma consecutiva y que se relacionan entre sí:

- La velocidad del automotor cada intervalo de lectura de datos a lo largo de los i-ésimos arcos, también Ilamada velocidad instantánea, esta se utiliza para establecer las variaciones de la velocidad en un arco en particular entre dos puntos, esta velocidad se obtiene mediante la aplicación de la ecuación (1).

$$
v_{i}=\frac{3.6}{t} \sqrt{\left(y_{2}-y_{1}\right)^{2}+\left(x_{2}-x_{1}\right)^{2}}
$$

Donde: $v_{i}=$ velocidad en $\mathrm{km} / \mathrm{h} ; x_{1}, y_{1}=$ coordenadas del punto 1 en metros; $x_{2}, y_{2}=$ coordenadas del punto 2 en metros; $t=$ intervalo de tiempo en segundos entre dato y dato.

- La velocidad promedio de operación del i-ésimo arco, la cual se obtiene mediante la relación entre la longitud del arco y la diferencia de los 
tiempos de paso entre el nodo inicial y el nodo final del mismo, aplicando la ecuación (2).

$$
v_{i}^{a}=3.6 \frac{l_{a}}{t_{2}-t_{1}}
$$

Donde: $v_{i}^{a}=$ velocidad $i$ en el $\operatorname{arco} a(\mathrm{~km} / \mathrm{h}) ; l_{a}$ $=$ longitud del arco $a$ en metros; $t_{1}=$ hora de paso en el nodo inicial; $t_{2}=$ hora de paso en el nodo final.

- La velocidad de operación para cada arco $i$ de una determinada ruta y en un período de tiempo dado; esta se calcula mediante la aplicación de la ecuación (3) y se emplea para establecer las impedancias de la red.

$$
\overline{v_{a}}=\frac{\sum_{i=1}^{n} v_{i}^{a}}{n}
$$

Donde: $\overline{v_{a}}=$ velocidad promedio de operación del arco $a ; n=$ número de datos de velocidad registrados en el arco a, para un período de tiempo.

La velocidad media de operación del flujo que se use es una variable fundamental para el análisis de accesibilidad (Herce \& Magrinya, 2002), independientemente de la escala de estudio, y de su consecución depende en gran parte la precisión y veracidad de los resultados que se obtengan.

\section{Cálculo de la accesibilidad media global o accesibilidad media integral}

Las isócronas son aquel espacio virtual conformado por las posiciones alcanzadas por un objeto en un tiempo Ti partiendo desde un punto $i$ en un arreglo radial, es decir, la red vial de la zona analizada. La accesibilidad media global se analiza a partir del valor de tiempo promedio de viaje (Tvi), el cual mide el tiempo promedio de viaje desde el nodo $i$ hasta los demás nodos de la red. Para su cálculo, se corre un algoritmo del SIG que permite calcular el menor tiempo de viaje entre un nodo específico y los demás nodos de la red, conformando una matriz unimodal de tiempos de viaje, la cual permite calcular el vector de tiempos medios de viaje, calculado mediante la aplicación de la ecuación (4).

$$
\overline{T_{v i}}=\frac{\sum_{j=1}^{m} t_{v i}}{(n-1)} \quad i=1,2,3, \ldots, n ; \quad j=1,2,3, \ldots, m
$$

Donde: $T v i=$ tiempo de viaje mínimo promedio entre el nodo $i$ y los demás nodos de la red; $t v i=$ tiempo de viaje mínimo entre el nodo $i$ y los demás nodos de la red; $n=$ número de nodos de la red.

El vector de tiempos medios de viaje obtenido se relaciona con las coordenadas geográficas (longitud y latitud), con el fin de aplicar el modelo geoestadístico de predicciones de tiempos medios de viaje en toda el área de estudio.

Ahora bien, para el cálculo de la accesibilidad media integral se debe definir con claridad el o los nodos desde los cuales se desea realizar el estudio, generándose el cálculo del vector de tiempo medio de viaje como se observó en la ecuación (4). La diferencia entre ambos tipos de accesibilidad es que la accesibilidad media global permite conocer la configuración de tiempos medios de viaje en un área en forma general, sin definir un punto o puntos geográficos con los cuales relacionar dichos tiempos medios de viaje, lo que sí ocurre en la accesibilidad media integral.

Un análisis de accesibilidad media global permite establecer impactos que producen las modificaciones a la red de infraestructuras en forma general, mientras que un análisis de accesibilidad media integral permite conocer el impacto que genera una modificación en la red de infraestructuras pero relacionada con un punto o unos puntos específicos de la red, como, por ejemplo, los nodos de actividad educativos.

\section{Comparación de accesibilidades y cálculo de coberturas}

A partir de las curvas isócronas de accesibilidad media global o integral es posible generar cruces 
de información entre las mismas e información de tipo sociodemográfico o socioeconómico, buscando conocer los valores de cobertura que se tendría por una curva de tiempo medio de viaje y su relación con variables como, por ejemplo, población, número de viviendas o simplemente área.

También es posible comparar las curvas isócronas obtenidas antes y después de una intervención infraestructural, con el fin de establecer las zonas que reportan un impacto, positivo o negativo, dada la inserción de la nueva infraestructura. El impacto es posible medirlo en términos de ahorro en tiempo medio de viaje o en términos de costos.

\section{EJEMPLOS A NIVEL REGIONAL}

A continuación se muestran dos ejemplos de aplicación de análisis de accesibilidad a nivel regional, el primero relacionado con la ubicación del futuro Aeropuerto del Café y el segundo relacionado con un análisis geoespacial que busca definir el mejor sitio de ubicación de una estación de gas natural comprimido.

\section{Región Vial Centro Sur de Caldas}

Dentro del proyecto "Estudios para el DesarroIlo del Ordenamiento Territorial y estudios técnicos para el sistema vial de la Región Centro Sur del Departamento de Caldas" se hizo el análisis de accesibilidad media global, ofrecida por la red viaria tanto actual como futura. La región centro - sur de Caldas tiene una superficie de 1.583 $\mathrm{km}^{2}$ (20,2\% del territorio departamental) alberga $54.8 \%$ del total de población departamental y posee una densidad poblacional de $338 \mathrm{hab} . / \mathrm{km}^{2}$, cubre los municipios de Manizales, Chinchiná, ViIlamaría, Neira y Palestina, siendo considerada la región más dinámica del departamento (Gobernación de Caldas, 2009). Para el análisis se definió un área de estudio de $3.848 \mathrm{~km}^{2}$, los cuales corresponden a una distribución radial $(r=35 \mathrm{~km})$ con centro en el casco urbano del municipio de
Palestina, donde está actualmente en construcción el Aeropuerto del Café.

La red vial de la Región Centro - Sur del Departamento de Caldas (575 arcos y 446 nodos) tiene una longitud total de $1.250 \mathrm{~km}$, de los cuales más de $56 \%$ pertenecen a tipos de vía primaria y secundaria, de forma semejante, más de $57 \%$ de la longitud refiere velocidades de operación inferiores a $30 \mathrm{~km} / \mathrm{h}$; no obstante, la totalidad de la red primaria reporta velocidades de operación superiores a $30 \mathrm{~km} / \mathrm{h}$. Se estableció que la velocidad promedio de la red es aproximadamente $26 \mathrm{~km} / \mathrm{h}$.

Las isócronas representan los sectores que podrían ser alcanzados en un tiempo promedio de viaje determinado, desde cualquier nodo de la red. Este tiempo de desplazamiento depende de las características físicas y operativas de la vía (topografía, estado de la superficie de rodadura, categoría, velocidad de operación promedio, sitios geotécnicos problemáticos, etc.) La curva isócrona de menor tiempo obtenida fue de 80 min., y la de mayor tiempo medio de viaje fue de 180 minutos, para la situación sin intervención.

Haciendo un comparativo entre la cobertura de las curvas isócronas con y sin intervención infraestructural, se obtuvo que los porcentajes de población cubierta sufren una mínima modificación, teniendo que para el rango de curvas isócronas de tiempo menores o iguales a $95 \mathrm{~min}$., el porcentaje de población cubierta es el mismo; para el rango de curvas isócronas de tiempo de entre 100 y 120 min., aumenta el porcentaje de cobertura de población 1\% aproximadamente y para el rango de curvas isócronas superiores a 120 min. el porcentaje disminuye en 1\%. Los anteriores resultados permiten concluir que las futuras intervenciones infraestructurales, desde el punto de vista de población cubierta por determinados tiempos de viaje, no impactarán significativamente el territorio.

No obstante, es posible obtener las curvas gradientes de accesibilidad media global, lo cual es simplemente la diferencia entre las curvas 
isócronas obtenidas con la red viaria actual y las obtenidas con las intervenciones infraestructurales. En la figura 1 se aprecian dichas curvas gradiente; en este caso, las isócronas representan las zonas que ganan un tiempo relativo de viaje, mostrando que estas causarán una disminución en el tiempo medio de viaje de entre 2.5 y 5 min.

Se tiene entonces que si el principal objetivo de las intervenciones viales que se realizarán es el de proveer mejoras relativas en los tiempos de desplazamiento en la región Centro Sur del Departamento de Caldas, es posible concluir que dicho objetivo se cumple, no obstante, la magnitud de dichas mejoras de tiempo no se pueden considerar sustanciales, así mismo es posible observar la necesidad latente de construir o mejorar la conexión entre la región Centro Sur y las poblaciones situadas hacia el norte del Departamento de Caldas, para garantizar una adecuada conexión de dichas áreas con el futuro Aeropuerto del Café.

\section{Distribución de gas natural comprimido, GNC}

En este caso se analiza la accesibilidad media global que ofrece la red vial del occidente caldense y del norte de Risaralda; así mismo, se analizan las accesibilidades integrales desde las poblaciones de Manizales, Chinchiná y La Virginia, con el fin de definir cuál de las tres poblaciones es la más adecuada para establecer la estación de distribución de GNC.

El análisis se complementa con el cálculo de las matrices de costo de transporte, entre las posibles poblaciones de distribución (La Virginia, Chinchiná y Manizales) y las poblaciones a servir a lo largo de los corredores viales definidos, en las cuales se aplica el camino mínimo de conexión entre nodos, minimizando la variable costos, para cada caso y para cada tipo de vehículo estudiado. Las matrices de costos así obtenidas, a partir de datos del INVÍAS, del Plan Vial de Caldas (Gobernación de

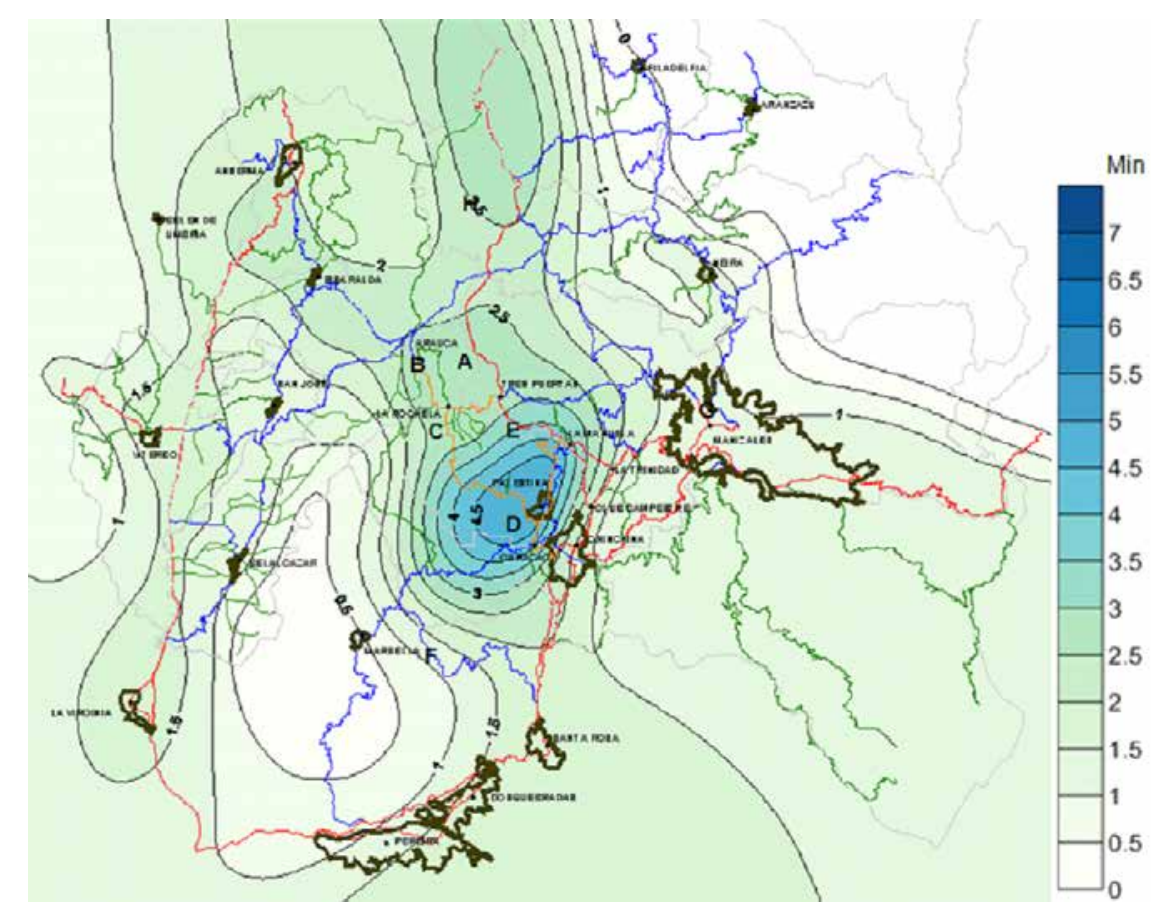

Figura 1. Curvas isócronas de accesibilidad media global para el gradiente de tiempo medio de viaje entre el escenario actual y futuro.

Fuente: Elaboración propia. 
Caldas, 2009) y de la aplicación del SIG, se comparan con la matriz de costos generada por la Comisión de Regulación de Energía y Gas, CREG.

Respecto a la variable tiempo medio de viaje, desde cada uno de los posibles orígenes de distribución, se obtuvieron curvas isócronas de accesibilidad integral según la variable tiempo de viaje y según el tipo de vehículo, lo cual permitió hacer una comparación entre matrices de viaje y obtener las curvas gradiente de accesibilidad integral entre cada posible origen. Se encuentra entonces que, según la variable tiempo medio de viaje en vehículo articulado o pesado, es óptimo distribuir desde Chinchiná, ya que se cubre una mayor área del territorio a servir con menores gradientes de tiempo, al ser comparado con los otros dos orígenes. Para cada uno de los posibles orígenes de distribución, se obtuvieron curvas según la variable costo del transporte y tipo de vehículo, lo cual permitió hacer una comparación entre matrices de costo y obtener las curvas gradiente en \$/ton entre cada par de orígenes. Fue posible establecer que desde el punto de vista de costos, el sitio más estratégico para la distribución corresponde al municipio de Chinchiná, tanto para vehículos articulados como para vehículos pesados. En la figura 2 se observa un ejemplo de las curvas obtenidas para el caso del análisis de costos.

No obstante lo anterior, conjugando las variables analizadas en términos de tiempo de viaje y costo (\$/ton) según el tipo de vehículos (pesado o articulado), se proponen dos alternativas, la primera consiste en definir dos puntos de distribución, uno situado en el municipio de Manizales que se encargue del abastecimiento de las poblaciones hacia el corredor norte mediante vehículos pesados y otro punto de distribución localizado en la ciudad de La Virginia encargado del abastecimiento de las poblaciones hacia el corredor occidente por medio de vehículos articulados; la segunda

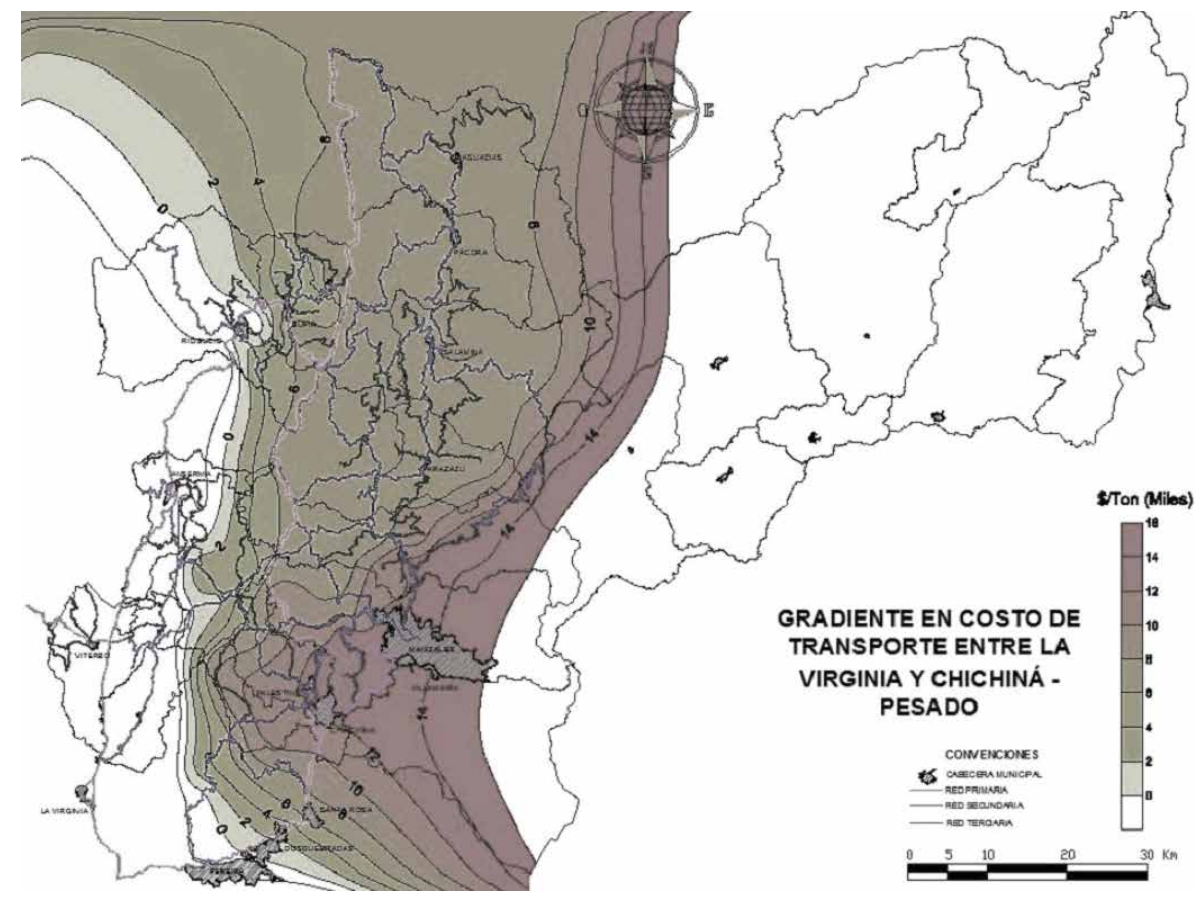

Figura 2. Gradiente en costo de transporte entre La Virginia y Chinchiná. Vehículo pesado.

Fuente: Elaboración propia. 
alternativa consiste en establecer una única estación de distribución en el municipio de Chinchiná, que se encargue del suministro del producto hacia los dos corredores analizados (corredor norte y corredor occidente), dadas sus condiciones de localización geográfica en la zona, así como sus características de infraestructura y conectividad.

En términos generales, las zonas más cercanas a los sitios de distribución pueden ser abastecidas mediante la programación de camiones pesados, en razón de que el gradiente de costos entre ambos tipos de vehículos es menor para distancias relativamente cortas. En la figura 3 se presentan los resultados encontrados al efectuar el análisis de costos de transporte por dos metodologías distintas, usando la matriz de costos de transporte del INVIAS (Instituto Nacional de Vías-INVIAS, 2009) y usando la matriz de costos del CREG (Comisión de Regulación de Energía y Gas-CREG, 2004) (Comisión de Regulación de Energía y Gas-CREG, 2005), las diferencias que presentan los resultados encontrados pueden deberse a diversos factores, como los siguientes:
- La infraestructura vial no es estática en el tiempo, siendo común que sea mayor el deterioro en el tiempo que la mejora de sus condiciones físicas, lo que guarda relación directa con los costos de operación vehicular.

- La matriz del CREG se actualiza con el IPC; sin embargo, este no es igual al incremento en la canasta del transporte, siendo generalmente esta última mayor que el IPC.

- En los últimos años han aparecido nuevas concesiones viales en el país que traen consigo la instalación de nuevos peajes, impactando también la estructura de costos.

\section{EJEMPLOS A NIVEL URBANO}

A continuación se muestra un ejemplo de aplicación de análisis de accesibilidad a nivel urbano, acerca de la relación entre la posición geoespacial de nodos de actividad y las características operativas de la red vial, lo cual se traduce en porcentajes de cobertura de un servicio determinado.

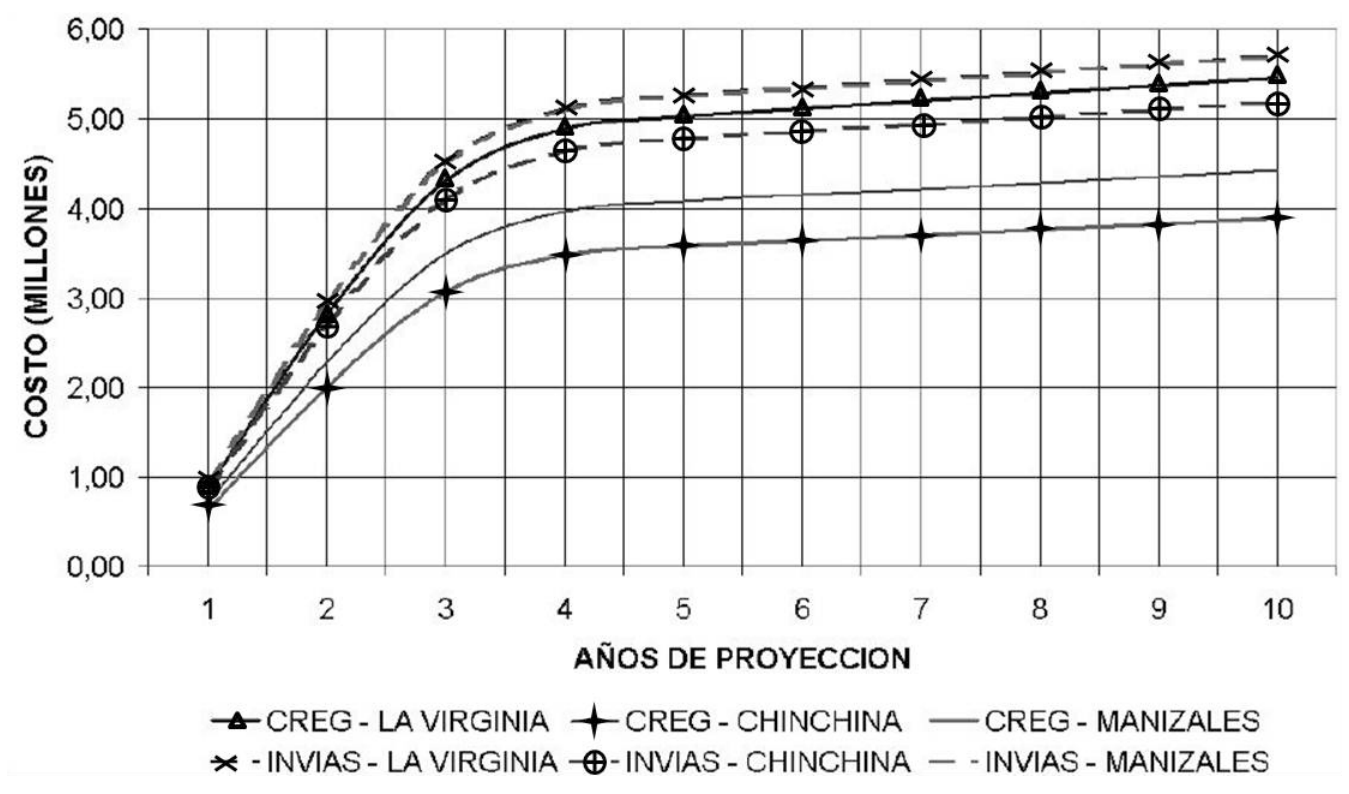

Figura 3. Comparación de costos matriz CREG y matriz INVIAS.

Fuente: Elaboración propia. 


\section{Cobertura de nodos de actividad}

En este caso se aplica análisis de accesibilidad con el objetivo de encontrar la relación existente entre las características operativas ofrecidas por la red de transporte de la ciudad de Manizales y la ubicación geoespacial de los centros educativos. La investigación se soporta en la toma de información primaria por un período superior a un año, con equipos GPS (más de 18 millones de datos). Se calculan los tiempos de desplazamiento que deben invertirse para alcanzar dichos nodos de actividad, así como las coberturas espaciales en términos de población y área. Se logra establecer en qué sectores de la ciudad existe una deficiencia respecto a la cobertura de estos nodos de actividad o cuáles de los centros educativos refieren deficiencia en su relación con los sistemas de transporte que llegan hasta cada uno de ellos.

Manizales refiere un total de 165 nodos de actividad educativa, de los cuales tres son de Ámbito Nacional, uno de Ámbito Regional y el resto (161) de Ámbito Local; todos los nodos educativos fueron debidamente georreferenciados en el SIG y a partir de dicha posición se hacen los cálculos de accesibilidad media integral. En la figura 4 se observan las curvas de tiempos medios óptimos e ideales de viaje que representan los tiempos que deben invertirse para alcanzar los nodos de actividad educativos. Se observa un amplio sector de la ciudad cubierto por la curva de 5 minutos, indicando que desde dichos sectores es posible alcanzar un nodo de actividad de tipo educativo si se invierte dicha cantidad de tiempo.

Los análisis de cobertura de las variables área, población y número de viviendas permiten establecer conclusiones respecto a los sectores en donde se concentra dicha actividad con relación a las características operativas de la red vial.

Se obtuvo que $55 \%$ del área, $72 \%$ de la población y $72 \%$ del número de viviendas de la ciudad podrían alcanzar un nodo de actividad educativo si se invierten hasta 5 minutos de tiempo medio de viaje. Se concluye que dadas las características de operación de la red vial actual, este tipo de

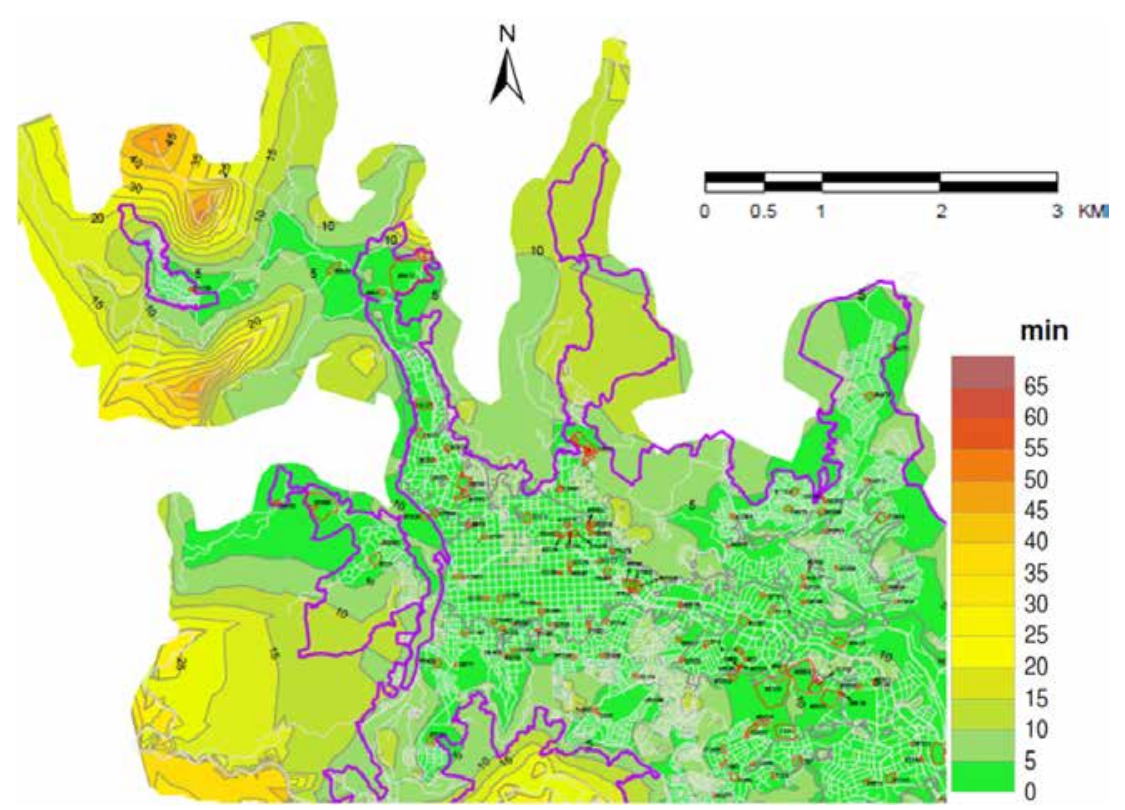

Figura 4. Tiempos medios óptimos e ideales de viaje hacia los nodos de actividad educativo.

Fuente: Elaboración propia. 
actividad refiere $100 \%$ de cobertura de las tres variables analizadas si se invierten aproximadamente hasta 15 minutos de tiempo medio de viaje.

En la figura 5 se observa la distribución de los porcentajes de cobertura de las variables área, población y número de viviendas cubiertas, según el tiempo medio de viaje. Se observa que la mayor cobertura se logra para tiempos medios óptimos e ideales de viaje de entre 0 y 5 minutos, con valores de cobertura superiores a $50 \%$.

Se hizo un análisis del porcentaje acumulado cubierto para la variable población según los diferentes nodos de actividad estudiados en el marco del Plan de Movilidad de Manizales, cuyos resultados se observan en la figura 6 . De modo general, se tiene que si una curva se encuentra más hacia la derecha del origen y tiene una menor pendiente, indica que para alcanzar dicho nodo de actividad se debe invertir un mayor tiempo medio de viaje. Se obtuvo, por ejemplo, que los nodos de actividad funerarias y recreativos son los que refieren curvas acumuladas de menor pendiente y desplazadas más hacia la derecha del origen, lo cual permite concluir que tienen una distribución geoespacial en la ciudad más desfavorable si se compara con los otros nodos de actividad; por su parte, para los nodos de actividad educativos y parques, las curvas acumuladas tienen una mayor pendiente y se ubican más cerca del origen, lo cual permite concluir que son dichos nodos de actividad los que presentan una mejor distribución geoespacial en la ciudad.

\section{CONCLUSIONES}

La metodología que abarca un análisis de accesibilidad, sea a escala regional o a escala urbana, se configura como una potente herramienta de diagnóstico y planificación de redes de movilidad, ya que permite conocer la relación existente entre las características operativas de un conjunto de modos de transporte y la infraestructura de movilidad por la cual discurren dichos modos. Con la aplicación de esta metodología se pueden alcanzar múltiples objetivos, entre los cuales se encuentran los siguientes:

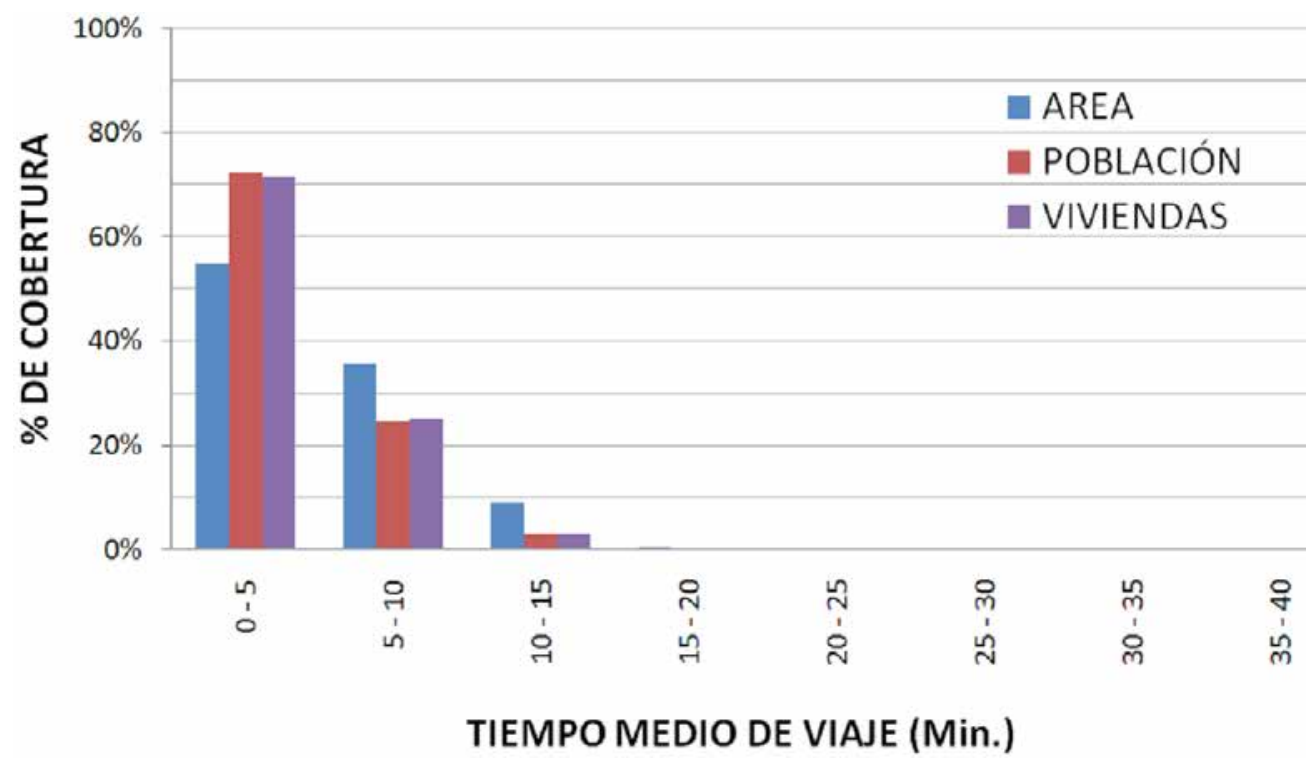

Figura 5. Distribuciones de los porcentajes de área, población y número de viviendas cubiertas por las curvas de tiempos medios óptimos e ideales de viaje para los nodos de actividad de tipo educativo.

Fuente: Elaboración propia. 


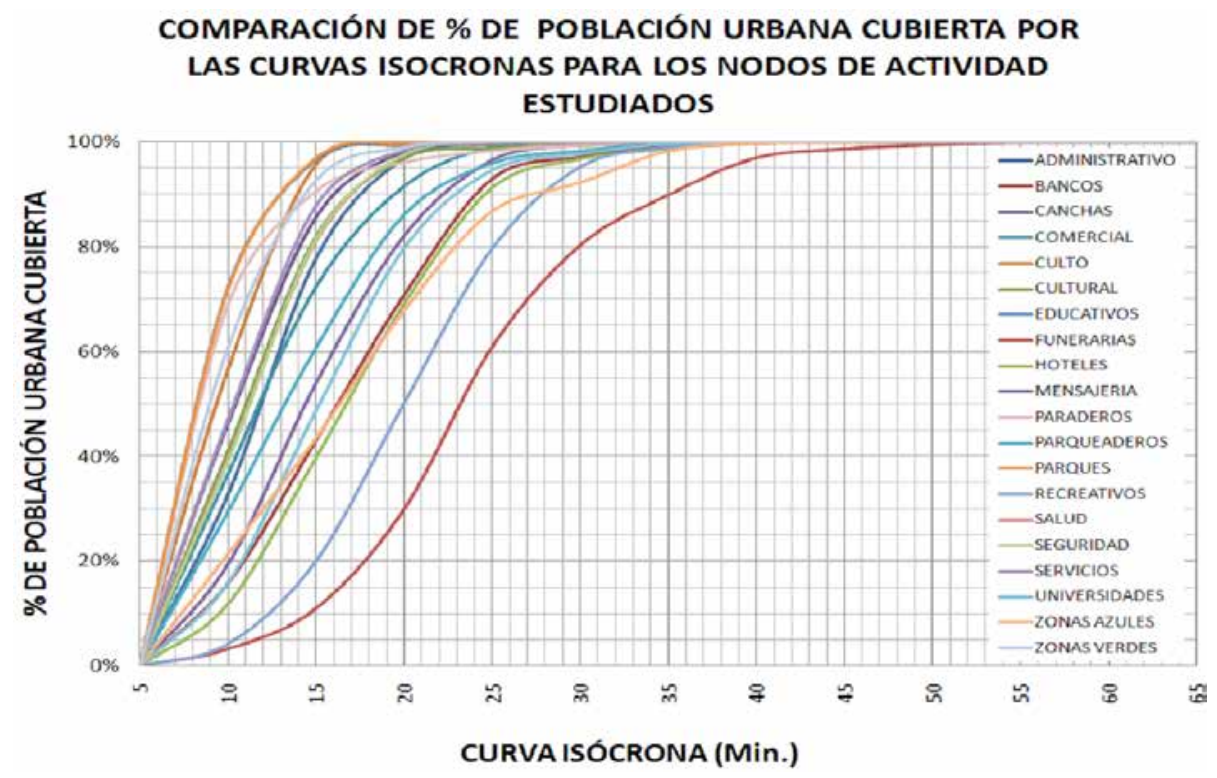

Figura 6. Comparación del porcentaje acumulado de población cubierta por las curvas isócronas para los diferentes nodos de actividad estudiados en la ciudad de Manizales.

Fuente: Elaboración propia.

- Actualización minuciosa de la red de infraestructuras del transporte del territorio objeto de análisis, lo cual demuestra las inmensas ventajas de combinar el uso de Sistemas de Información Geográfica con tecnologías de monitorización satelital (GPS).

- Diagnóstico de la localización geoespacial de los diferentes nodos de actividad que se encuentran en un territorio y análisis de su cobertura sociodemográfica.

- Estudio de la relación entre ubicación geoespacial de nodos de actividad y tiempos de respuesta de variables asociadas a dichos nodos; por ejemplo, nodos relacionados con seguridad o salud en una urbe, lo cual permitiría identificar las áreas del territorio que reportan ausencia de cierto nodo de actividad.

- Identificación de zonas del territorio que refieren un mayor desequilibrio en los tiempos medios de viaje o en el ofrecimiento de cierto servicio.

- Determinación del impacto generado por la construcción de cualquier tipo de infraestructura del transporte, o evaluación del impacto dados los cambios propuestos en la gestión de un modo de transporte en particular.

En Colombia la metodología ha sido poco aplicada; no obstante, se demuestra con estas investigaciones que esta se convierte en un apoyo técnico que muestra un panorama general de las condiciones de accesibilidad de un territorio que en cualquier momento puede soportar la toma de decisiones respecto a modificaciones que se desee hacer a la red de infraestructuras del transporte o a los sistemas de transporte como tal. La metodología permite establecer con claridad en qué áreas de un territorio específico se deben aunar esfuerzos para ofrecer una mejor accesibilidad y aumentar la calidad de vida de los pobladores, todo esto relacionado con los diferentes usos del suelo y nodos de actividad presentes en un área determinada.

\section{FINANCIAMIENTO}

Universidad Nacional de Colombia, Sede Manizales 


\section{REFERENCIAS}

Batty, M. (2009). Accessibility: in search of a unified theory. Environment and Planning B: Planning and Design, 36(2), 191-194.

Burkey, M. (2012). Decomposing geographic accessibility into component parts: methods and an application to hospitals. Annals of Regional science Forthcoming, 48(3), 783-800.

Calcuttawala, Z. (2006). Landscapes of Information and Consumption: A Location Analysis of Public Libraries in Calcutta (E. Garten, D. Williams, \& J. Nyce, eds.) Advances in Library Administration and Organization, 24, 319-388.

Cheng, J.; Bertoliniand, L., \& Clercq, F. (2007). Measuring Sustainable Accessibility. Transportation research Board: Journal of the Transportation Research Board, 2017, 16-25.

Comisión de Regulación de Energía y Gas, CREG (2004). Compresión y transporte de gas natural comprimido-GNC. Propuesta regulatoria para consulta-048. Bogotá.

Comisión de Regulación de Energía y Gas, CREG (2005). Resolución 008 de 2005. Bogotá.

Curl, A.; Nelson, J., \& Anable, J. (2011). Does Accessibility Planning address what matters? Transportation Business \& Management, 2, 3-11.

Escobar, D., \& García, F. (2012). Análisis de accesibilidad a nodos de actividad en Manizales (Colombia). Manizales: Universidad Nacional de Colombia, Sede Manizales. Facultad de Ingeniería y Arquitectura.

Escobar, D., \& García, F. (2012). Análisis de Priorización de proyectos viales. Manizales: Universidad Nacional de Colombia, Sede Manizales. Facultad de Ingeniería y Arquitectura.

Escobar, D., \& García, F. (2012). Diagnóstico de la movilidad urbana de Manizales. Manizales: Universidad Nacional de Colombia, Sede Manizales.

Escobar, D., \& García, F. (2012). Territorial Accessibility Analysis as a Key Variable for Diagnosis of Urban Mobility: A case study. Manizales (Colombia). Procedia-Social and Behavioral Sciences.
Transporte Research Arena. 48, pp. 1385-1394. Athens: Elsevier.

Escobar, D.; García, F., \& Tolosa, R. (2013). Análisis de accesibilidad territorial a nivel regional. Manizales: Universidad Nacional de Colombia, Sede Manizales. Facultad de Ingeniería y Arquitectura.

Farrington, J., \& Farrington, C. (2005). Rural accessibility, social inclusion and social justice: towards conceptualisation. Journal of Transport Geography, 13(1), 1-12.

Gobernación de Caldas (2009). Plan Vial de Caldas 2008-2017. Manizales.

Gutierrez, J. (1998). Redes, espacio y tiempo. Anales de Geografía de la Universidad Complutense (18), 65-86.

Gutiérrez, J., \& Gómez, G. (1999). The impact of orbital motorways on intra-metropolitan accessibility: the case of Madrid's M-40. Journal of Transport Geography, 7(1), 1-15.

Gutiérrez, J., \& Gómez, G. (1999). The impact of orbital motorways on intra-metropolitan accessibility: the case of Madrid's M-40. Journal of Transport Geography, 7(1), 1-15.

Gutiérrez, J.; Condeco-Melhorado, A., \& Martin, J. (2012). Using Accessibility indicators and GIS to assess spatial spillovers of transport infrastructure investment. Journal of Transport Geography, 18(1), 141-152.

Gutiérrez, J.; Condeco-Melhorado, A., \& Martin, J. (2012). Using Accessibility indicators and GIS to assess spatial spillovers of transport infrastruture investments. Journal of Transport Geography, 18(1), 141-152.

Halden, D. (2011). The use and abuse of accessibility measures in UK passenger transport planning. Transportation Business \& Management, 2, 12-19.

Herce, M., \& Magrinya, F. (2002). La Ingeniería en la evolución de la Urbanística. Barcelona: Ediciones UPC.

Higgs, G.; Langford, M., \& Fry, R. (2013). Investigating variations in the provision of digital services in public libraries using network-based GIS models. $\mathrm{Li}$ brary \& Information Science Research, 35(1), 24-32. 
Instituto Nacional de Vías, INVIAS (2009). Volúmenes de Tránsito 2007. Bogotá.

Jones, P. (2011). Developing and applying interactive visual tools to enhance stakeholder engagement in accessibility planning for mobility disadvantaged groups. Transportation Business \& Management, 2, 29-41.

Kastenholz, E.; Eusébio, C.; Figueiredo, E., \& Lima, J. (2012). Accessibility as a competitive advantage of a tourist destination-the case of Lousã. (K. Hyde, C. Ryan, \& A. Woodside, eds.) Emerald Publishers.

Kibambe, Lubamba, J.P.; Radoux, J., \& Defourny, P. (2013). Multimodal accessibility modeling from coarse transportation networks in Africa. International Journal of Geographical Information Science, 27(5), 1005-1022.

Kotavaara, O.; Antikainenand, H., \& Rusanen, J. (2011). Population change and accessibility by road and rail networks: GIS and statistical approach to Finland 1970-2007. Journal of Transport Geography, 19(4), 926-935.

López, E.; Gutiérrez, J., \& Gómez, G. (2008). Measuring regional cohesion effects of large-scale transport infrastructure investments: an accessibility approach. European Planning Studies, 16(2), 277-301.

Mackinnon, D.; Pirie, G., \& Gather, M. (2008). Transport and economic development. (R. Knowles, J. Shaw, \& I. Docherty, eds.) Transport Geographies: Mobilities, Flows and Spaces, 10-28.

Morris, J.; Dumble, P., \& Wigan, M. (1978). Accessibility indicators for transport planning. Transportation Research, A, 13(A), 91-109.
Park, S. J. (2012). Measuring public library accessibility: A case study using GIS. Library \& Information Science Research, 34(1), 13-21.

Rietveld, P., \& Nijkamp, P. (1993). Transport and regional development. En J. Polak, \& A. Heertje (ed.), European Conference of Ministers of Transport (ECMT). Oxford: Blackwell Publishers.

Sailer, K.; Marmot, A., \& Penn, A. (2012). Spatial Configuration, Organisational Change and Academic Networks. Conference for 'Applied Social Network Analysis. Zurich.

Schürmann, C.; Spiekermann, K., \& Wegener, M. (1997). Accessibility indicators. Berichte aus dem Institut für Raumplanung, 39.

Straatemeier, T. (2008). How to plan for regional accessibility? Transport Policy, 15, 127-137.

Vega, A. (2011). A multi-modal approach to sustainable accessibility in Galway. Regional Insights, 2(2), 15-17.

Vickerman, R.; Spiekermann, K., \& Wegener, M. (1999). Accessibility and economic development in Europe. Regional Studies, 33(1), 1-15.

Wang, D.; Brown, G., \& Mateo-Babiano, D. (2013). Beyond proximity: an integrated model of accessibility for public parks. Asian Journal of Social Sciences \& Humanities, 2(3), 486-498.

Zhu, X., \& Liu, S. (2004). Analysis of the impact of the MRT system on accessibility in Singapore using an integrated GIS tool. Journal of Transport Geography, 12(2), 89-101.

\section{(c) $(1) \Theta \Theta$}


